\title{
Neuroinflammation and Neutrophils: Modulation by Ouabain
}

\author{
Jacqueline Alves Leite ${ }^{1 \dagger}$, Luiz Henrique Agra Cavalcante-Silva ${ }^{2+}$, Martina Raissa Ribeiro ${ }^{3}$, \\ Geovanni de Morais Lima $^{3}$, Cristoforo Scavone ${ }^{3 *}$ and Sandra Rodrigues-Mascarenhas ${ }^{2}$ \\ ${ }^{1}$ Department of Pharmacology, Institute of Biomedical Science, Federal University of Goiás, Goiânia, Brazil, \\ ${ }^{2}$ Immunobiotechnology Laboratory, Biotechnology Center, Federal University of Paraíba, João Pessoa, Brazil, ${ }^{3}$ Laboratory of \\ Molecular Neuropharmacology, Department of Pharmacology, Institute of Biomedical Science, University of São Paulo, São \\ Paulo, Brazil
}

Cardiotonic steroids are natural compounds that present many physiological and pharmacological functions. They bind $\mathrm{Na}^{+} / \mathrm{K}^{+}$-ATPase (NKA) modifying cellular ion concentration and trigger cell signaling mechanisms without altering ion balance. These steroids are known to modulate some immune responses, including cytokine production, neutrophil migration, and inflammation (peripherally and in the nervous system). Inflammation can occur in response to homeostasis perturbations and is related to the development of many diseases, including immune-mediated diseases and neurodegenerative disorders. Considering the neutrophils role in the general neuroinflammatory response and that these cells can be modulated by cardiac steroids, this work aims to review the possible regulation of neutrophilic neuroinflammation by the cardiac steroid ouabain.

Keywords: innate immunity, inflammation, ouabain, neuroimmune interactions, neuropharmacology

\section{OUABAIN AND NA ${ }^{+} / \mathrm{K}^{+}$-ATPASE} criscavone@usp.br

${ }^{\dagger}$ These authors have contributed equally to this work and share first authorship

Specialty section: This article was submitted to Inflammation Pharmacology,

a section of the journal

Frontiers in Pharmacology

Received: 29 November 2021 Accepted: 10 January 2022

Published: 31 January 2022

Citation:

Leite JA, Cavalcante-Silva LHA Ribeiro MR, de Morais Lima G, Scavone $C$ and

Rodrigues-Mascarenhas S (2022) Neuroinflammation and Neutrophils:

Modulation by Ouabain.

Front. Pharmacol. 13:824907.

doi: 10.3389/fphar.2022.824907
Ouabain is a cardiotonic steroid identified by Hamlyn et al. (1991) in mammalian plasma. Studies have shown that ouabain can be produced by the adrenal gland, hypothalamus, and pituitary, being considered a hormone (Pamnani et al., 1981; Hamlyn et al., 1991; Ferrandi et al., 1997). In relation to the adrenal, ouabain synthesis appears to occur in the glomerulosa of the cortex (Laredo et al., 1995), and its release can be stimulated by two different hormones, angiotensin II, or adrenocorticotropic hormone (Laredo et al., 1994). The physiological levels of circulating ouabain in humans are approximately $0.2 \mathrm{nM}$ and, in rodents, this value can reach $0.5 \mathrm{nM}$ (Blaustein and Hamlyn, 2020).

Its receptor is the NKA, being the only established receptor for cardiotonic steroids such as ouabain, which interacts with amino acids located in the extracellular loops of the a subunit of the enzyme (Dvela et al., 2007). NKA is a membrane protein responsible for establishing and maintaining high $\mathrm{K}^{+}$and low $\mathrm{Na}^{+}$concentrations in the intracellular environment, in addition to maintaining cellular osmotic balance, the resting potential of most body tissues and the properties of excitable muscle and neural cells (Blanco and Mercer, 1998). The enzyme is composed of the $a$ subunit, responsible for the catalytic activity and ion transport function, and the $\beta$ subunit, which is necessary for the enzyme activity, regulating the fixation of the a subunit, and modulating the affinity for $\mathrm{Na}^{+}$and $\mathrm{K}^{+}$ions. There are four $\alpha$ isoforms, with $\alpha 1$ being ubiquitously expressed, while the $\alpha 2-a 4$ isoforms present a more restricted cellular distribution (Markov et al., 2020). The modulating actions of this receptor affect both the cellular ionic balance, changing different cellular functions, such as cell migration (Ward and Becker, 1970), but also as an important signal transducer (Liu and 
Xie, 2010; Fan et al., 2017). At low concentrations (usually at nanomolar range), ouabain can promote conformational changes in NKA, without inhibiting the transport of sodium and potassium (Liu et al., 2000; Xie, 2003). This process leads to the activation/inhibition of many cell signaling proteins,

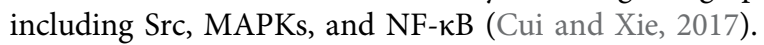

Therapeutically, cardiotonic steroids are usually recommended for congestive heart failure, but only digoxin remains in use (Alpert, 2021). Despite this, ouabain presents many physiological and pharmacological effects that are more studied in the cardiovascular system, in the renal and brain tissues (reviewed in Blaustein and Hamlyn, 2020 and Bagrov et al., 2009). Other authors also suggest a repurposing of cardiac glycosides, including ouabain, for cancer therapeutics (Schneider et al., 2017; Matozzo et al., 2020; Du et al., 2021). Cardiac glycosides also modulate inflammation and autoimmune diseases (Škubník et al., 2021). Digoxin can inhibit transcriptional factor ROR $\gamma$ t, thus inhibiting Th17 cells, a cell type involved in autoimmunity (Huh et al., 2011). Another cardiac glycoside, bufalin, inhibits the allergic inflammation by suppression of nuclear factor-kappa B activity (Zhakeer et al., 2017).

Regarding ouabain, many immune system cells can be modulated (Olej et al., 1994; Rodrigues-Mascarenhas et al., 2009). In thymocytes, precursor cells of $\mathrm{T}$ lymphocytes, ouabain inhibits cell proliferation (Szamel et al., 1981), induces the expression of CD69 (Rodrigues Mascarenhas et al., 2003) and the increase of free radicals (Smolyaninova et al., 2013). Also, in thymocytes, ouabain also reduces the activation of the mitogenactivated protein kinase (MAPK) p38 and the levels of the nuclear activating factor of T cells c1 (NFAT1c) (Rodrigues-Mascarenhas et al., 2008). Ouabain also inhibits lymphocyte mitosis. Furthermore, it was also evidenced that ouabain negatively modulates the number of B lymphocytes in the bone marrow, spleen, and peripheral blood (de Paiva et al., 2011), without, however, changing the levels of immunoglobulin $\mathrm{G}(\mathrm{IgG})$ and $\mathrm{M}$ (IgM). On the other hand, ouabain induces an increase in the number of B lymphocytes in the mesenteric lymph nodes, probably due to the reduction in the expression of the adhesion molecule CD62L and the chemokine receptor CXCR5 (da Silva et al., 2016).

Other immune system cells can also be modulated by ouabain. Nascimento et al. (2014) demonstrated that ouabain regulates the maturation of dendritic cells. In human monocytes, ouabain negatively regulates the expression of $\mathrm{mCD} 14$, a cell surface molecule involved in the response against Gram-negative bacteria, through the activation of the epidermal growth factor receptor (EGRF) and MAPK p38 (Valente et al., 2009). In addition, monocytes treated with ouabain have high levels of CD69, HLA DR, CD86, and CD80, molecules related to cell activation, in addition to increasing the phagocytic capacity of these cells (Teixeira and Rumjanek, 2014). Ouabain also inhibits, in vitro, the development of an inflammatory monocyte subtype $\left(\mathrm{mCD}_{14}{ }^{+} \mathrm{CD} 16^{+}\right)$(Valente et al., 2009) and stimulates the production of cytokines such as IL- $1 \alpha$, IL- $1 \beta$, TNF- $\alpha$, IL- 6 and IL-10 (Foey et al., 1997; Matsumori et al., 1997; Teixeira and Rumjanek, 2014).
Moreover, ouabain can modulate inflammation and inflammatory cells (reviewed in Cavalcante-Silva et al., 2017). Dysregulated migration or activation of inflammatory cells, such as neutrophils are involved in the immunopathogenesis of many diseases (Leliefeld et al., 2016; Hellebrekers et al., 2018; Cavalcante-Silva et al., 2021b; Bautista-Becerril et al., 2021; Parackova et al., 2021). Thus, modulating these cells can be a therapeutic approach for inflammatory diseases. The ouabain effect on neutrophil during peripheral and neuro inflammation will be discussed below.

\section{NEUROINFLAMMATION AND NEUTROPHILS}

For a long time, the central nervous system (CNS) was recognized as a "privileged immune" organ due to the presence of the bloodbrain barrier (BBB), which was previously considered to be almost impenetrable. Nevertheless, several studies have pointed out the flexibility in the BBB in response to inflammatory stimuli, which can generate a process known as neuroinflammation (Kanashiro et al., 2020). Neuroinflammation is related to the emergence of neurodegenerative diseases, such as Parkinson's disease, Alzheimer's disease (AD) and cerebral ischemia as well as its association with neuropsychiatric disorders. Thus, neuroinflammation has been studied as an important therapeutic target for the treatment of neurodegenerative and neuropsychiatric diseases (Akira et al., 2006).

Inflammation in the CNS is orchestrated by resident immune cells such as astrocytes and microglia (Doty et al., 2015), as well as by the migration of monocytes and lymphocytes through the BBB. (Hawkes and McLaurin, 2009; Lampron et al., 2013). Several studies have pointed out the relevance of neutrophils in chronic neuroinflammatory diseases such as $\mathrm{AD}$, but their role still needs to be better elucidated. Neutrophil recruitment can cause neuronal damage and cognitive decline in $\mathrm{AD}$; however, some granular proteins, such as CAP37, neutrophil elastase and cathepsin $G$ can promote $A \beta$ cleavage, facilitating $A \beta$ clearance, preventing the formation of pathogenic aggregates (reviewed in Stock et al., 2018).

CNS cells, such as astrocytes, release several chemokines dependent on the IL-17 pathway, such as CXCL5, CXCL2, and CXCL1, which promote neutrophil migration to the CNS.

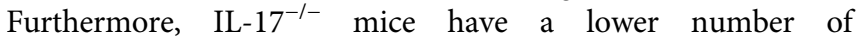
neutrophils infiltrating the CNS, but the same was not observed in the spinal cord, as neutrophil migration to the spinal cord appears to be regulated by IFN- $\gamma$ (Christy et al., 2013; Simmons et al., 2014; Pierson et al., 2018). On the other hand, in chronic neurodegenerative diseases such as $\mathrm{AD}$, neutrophil migration appears to be orchestrated by the chemokines CXCL12 and CCL2 as well as by the LFA-1 integrin (Zenaro et al., 2015). Interestingly, studies in mice have shown that blocking the LFA-1 integrin resulted in the inhibition of neutrophil migration, as well as the activation of microglia, resulting in reduced cognitive deficit in $\mathrm{AD}$ (Zenaro et al., 2015). 
Neutrophil infiltration in post-ischemic inflammation has been associated with exacerbation of brain injury and neuronal damage due to release of pro-inflammatory cytokines, production of reactive oxygen species and reactive nitrogen species, and (neutrophil extracellular traps) NETs release. Furthermore, these neutrophils have a hypersegmented characteristic and their migration is dependent on the CXCR2 neutrophil-specific chemokine receptor (Herz et al., 2015; Neumann et al., 2015). Additionally, it was observed that blocking through a neutralizing antiserum or selective pharmacological inhibitor for CXCR2 prevented the recruitment of neutrophils to the brain of hyperlipidemic mice ApoE $^{-/-}$mice (Herz et al., 2015). Furthermore, it was observed that polymorphonuclear cells develop direct neurotoxicity through the secretion of TNF-alpha, matrix metalloproteinase 9, as well as through heterocellular contact, which may thus contribute to secondary damage after brain injury, such as cognitive impairment (Dinkel et al., 2004; Nguyen et al., 2007). Another important point observed in spinal cord injury models is the participation of NF- $\kappa \mathrm{B}$ signaling in neutrophil activation and infiltration, since I $\mathrm{B}$ kinase (IKK)- $\beta$ conditional knockout mice showed lower secretion of CXCL1 and the consequent neutrophil infiltration resulted in less neuronal damage, neuroinflammation and improvement in motor function (Kang et al., 2011). Furthermore, Zenaro et al. showed that neutrophil infiltration plays an important role in microglia activation, accumulation of abnormal $A \beta$ and tau, synaptic dysfunction, and memory decline in the neuroinflammation observed in AD (Zenaro et al., 2015).

On the other hand, the neuroprotective role for neutrophils in post-ischemic inflammation has been observed by different works. In an in vitro study conducted by Hou et al. (2019) it was observed that while N1 neutrophils decreased neuronal viability, $\mathrm{N} 2$ polarization promoted an improvement in neuronal viability against oxygen glucosedeprivation/reoxygenation-induced injury, in cultured cortical neurons (Hou et al., 2019). In addition, using an in vivo model of injury induced by transient occlusion of the middle cerebral artery, Hou et al. (2019) showed that rats spontaneously regenerate over time, and that there is a negative correlation between the proportion of N2 neutrophils and the number of degenerating neurons, in the ipsilateral brain parenchyma (Hou et al., 2019). Furthermore, a study conducted in mice demonstrated that rosiglitazone, a peroxisome proliferator-activated receptor- $\gamma$ (PPARy) agonist, increased the infiltration of N2-type neutrophils, culminating in a neuroprotective effect after stroke. Interestingly, the neuroprotective effect of the PPAR $\gamma$ agonist was reversed after neutrophil depletion, demonstrating their importance in neuroprotection (Cuartero et al., 2013). In another study, García-Culebras et al. (2019) demonstrated that mice deficient in TLR4 have a greater polarization for the $\mathrm{N} 2$ profile, resulting in a smaller infarct volume and neuroprotection, elucidating a role of TLR4 signaling for neutrophil polarization during the cerebral ischemia (García-Culebras et al., 2019). In addition, Sas et al. showed a CD14 ${ }^{+}$Ly6G $^{\text {low }}$ granulocyte with features of an immature neutrophil with neuroregenerative and neuroprotective properties in models of optic nerve and spinal cord injury, resulting from the secretion of NGF and IGF-1 growth factors by CD $14^{+}$Ly6G $^{\text {low }}$ cells (Sas et al., 2020).

The different findings indicate that neutrophils are highly responsive to CNS lesions and may influence the process of neuroinflammation and neurodegeneration, as well as neuroprotection, affecting the development of neurodegenerative processes. Considering the development of neuroinflammation, ouabain emerge as a possible player in the regulation of this process (Figure 1A), as several studies have demonstrated the importance of digitalis and NKA in neuroinflammatory regulation (reviewed in Orellana et al., 2016).

Studies have demonstrated an in vivo and in vitro neuroprotective activity of ouabain. Kinoshita et al. (2014) have observed in vivo that ouabain has a protective effect against LPS-induced neuroinflammation in the rat hippocampus, through a reduction in the activation and consequent translocation of nuclear factor kappa B (NF- $\kappa \mathrm{B})$, leading to reduction in the expression of iNOS and IL-1 $\beta$. Furthermore, it was observed that the administration of ouabain reduced the activation of astrocytes, in the dentate gyrus, through a reduction in the expression of glial fibrillary acidic protein (GFAP) (Kinoshita et al., 2014). In addition, in vitro studies of LPS-stimulated astrocytes found that treatment with ouabain reduced the release of IL-1 $\beta$ (Forshammar et al., 2011). On the other hand, an in vitro study using LPS-stimulated rat microglial cell culture, it was shown that treatment with Ouabain did not alter the release of TNF- $\alpha$ and IL- $1 \beta$, thus suggesting a lack of modulating effect of ouabain on this cell type (Forshammar et al., 2013). Additionally, in a recently published study, Mázala-de-Oliveira et al. (2021) have shown that nanomolar concentrations of ouabain reduced the expression of inflammatory receptors, such as TNFR1, TLR4 and CD14 of rat retinal ganglion cells culture after optic nerve axotomy, in all tested periods. It was also observed that ouabain produced an increased survival of retinal ganglion cells after $48 \mathrm{~h}$, and the mechanism was dependent on autophagy, since the use of 3-methyladenine, an autophagy inhibitor, lead to a complete inhibition of the neuroprotective role of ouabain (Mázala-deOliveira et al., 2021).

On the other hand, it was shown that a2-NKA knockdown in superoxide dismutase 1 (SOD1) mutant astrocytes protected motor neurons from degeneration (Gallardo et al., 2014). Furthermore, it was observed that the silencing of a 2 -NKA in primary culture of glial cells from mice promoted a lower responsiveness to the stimulus with LPS, by reducing the production of TNF- $\alpha$, as well as the activation of ERK and $\mathrm{NF}-\mathrm{kB}$, suggesting the participation of $\alpha 2-\mathrm{NKA}$ in the modulation of LPS-induced neuroinflammation (Kinoshita et al., 2017). Ouabain, in vitro, protected motor neurons from degeneration induced by mutant SOD1 astrocytes (Gallardo et al., 2014). Heterozygous KI mice, carrying the G301R disease mutation $\left(a 2^{+/ G 301 R}\right.$ mice), exhibited familial hemiplegic migraine type 2 (FHM2)-related phenotypes, including mood depression and obsessive-compulsive disorder (OCD)-like symptoms (Isaksen and Lykke-Hartmann, 2016). Surprisingly, when subjected to spinal cord injury, $\alpha 2^{+/ G 301 R}$ mice show better 


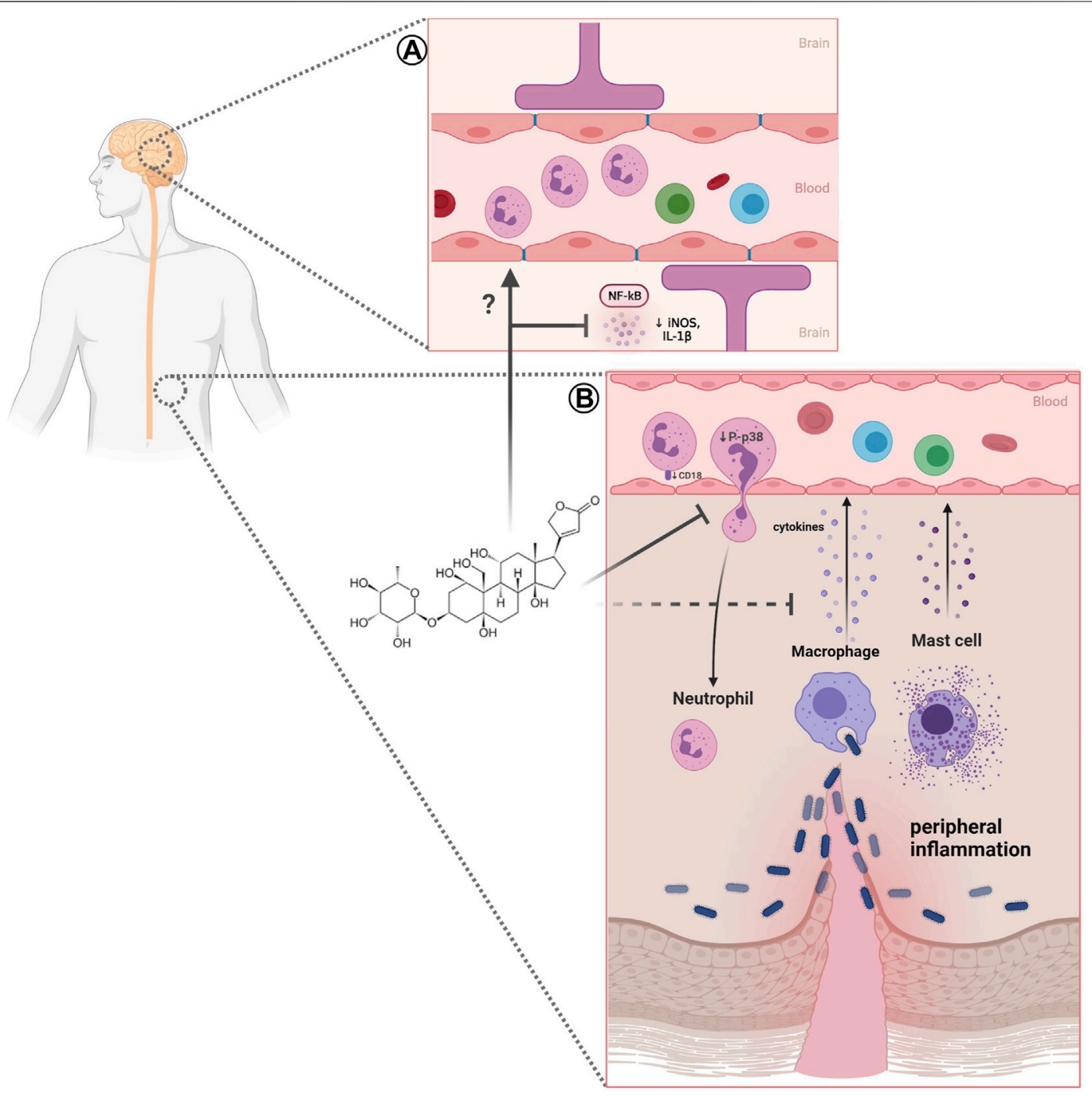

FIGURE 1 | Effect of ouabain on neutrophils in peripheral and neuroinflammation. (A) In neuroinflammation ouabain can reduces NF-kB and iNOS activation and inflammatory cytokines (IL-1), however, the effects of this steroid in neutrophils remains to be elucidated. (B) Impairment of neutrophil migration caused by ouabain may involve reduction of adhesion molecule CD18, P-p38 MAPK, and decrease of inflammatory cytokines. Created with BioRender.com.

functional recovery and decreased lesion volume compared to littered controls $\left(\alpha 2^{+/+}\right)$. These phenotypes were associated with alterations in pro and anti-inflammatory cytokines levels, such as IL-6, TNF, and IL-10 (Ellman et al., 2017). Furthermore, it was observed that $\alpha 2^{+/ G 301 R}$ mice showed a reduction in the systemic levels of the proinflammatory cytokines TNF- $\alpha$, IL- 6 and IL- $1 \beta$ after LPS administration, as well as a reduction in the hypothermic and neuroinflammatory response in the hippocampus and hypothalamus (Leite et al., 2020).

Additionally, peripheral inflammation can also be modulated by ouabain. This steroid can alter vascular permeability induced by different inflammatory agents. In the sheep skin and pleural cavity, ouabain reduces vascular permeability caused by turpentine, an irritant agent (Lancaster and Vegad, 1967). Additionally, in mice peritoneal cavity this steroid decreases vascular permeability induced by zymosan, a fungal wall component (Leite et al., 2015). Also in mice, ouabain can inhibits paw edema, a cardinal signal resulting of increased vascular permeability, induced by carrageenan, compound 48/ 80, zymosan, prostaglandin E2, and bradykinin (de Vasconcelos et al., 2011). This effect of ouabain in vascular parameters could be related to its effects on histamine (Okazaki et al., 1976) and/or cytokines release (Leite et al., 2015). Indeed, it has been reported that ouabain decreases levels of cytokines TNF- $\alpha$, IL-1 $\beta$ (Leite et al., 2015), and IFN- $\gamma$ (Jacob et al., 2013) in peritoneal inflammation.

It is noteworthy that many studies related that ouabain possesses a proinflammatory effect and facilitates immune cell migration (Kennedy et al., 2013; Gonçalves-de-Albuquerque et al., 2014; Chen et al., 2017). The possible dual effect of 
ouabain on inflammation may be due to different animal species studied (i.e., BALB/c, Swiss, or C57BL/6 mice; rats; humans) since it could impact NKA sensitivity to ouabain. Moreover, administration route used, presence of a previous inflammatory stimulus, or even different concentrations of this steroid leads to different outcomes. In fact, high levels of ouabain could cause an immune system activation and promote a pathological inflammatory response (Blaustein and Hamlyn, 2020).

\section{NEUTROPHILS: MODULATION BY OUABAIN}

During inflammation, endothelial cell activation by cytokines induces vascular permeability and enables migration of immune cells into tissues. Usually, neutrophils are the first immune cell to reach inflamed tissue (Liew and Kubes, 2019). Neutrophils are polymorphonuclear segmented cells with antimicrobial properties (Burn et al., 2021). However, these cells present many other immune functions and participate in the stimulation of adaptive immune responses (Minns et al., 2019), in the resolution of inflammation (Jones et al., 2016) and healing (Phillipson and Kubes, 2019), and have anti or pro-tumor activity (Mishalian et al., 2017; Ocana et al., 2017; Burn et al., 2021). Outside of neutrophils' essential role in immune system homeostasis, they are also involved in autoimmune and inflammatory diseases, such as arthritis (O'Neil and Kaplan, 2019) and COVID-19 (Cavalcante-Silva et al., 2021b). In these pathological conditions, neutrophils may have a dysregulated migration or activation (Hidalgo et al., 2019).

Neutrophil migration requires the interaction between adhesion molecules present on neutrophils and vascular endothelium (Nourshargh and Alon, 2014). Classic neutrophil recruitment involves different steps such as capture, rolling, adhesion, crawling, and subsequent transmigration towards inflammatory signals (Kolaczkowska and Kubes, 2013). Several works have demonstrated the usually low concentrations of ouabain impairs mice neutrophil migration into different tissues. Leite et al. (2015) showed that ouabain reduces neutrophil migration into the peritoneal cavity induced zymosan. Similar findings were obtained using L. amazonensis as an inflammatory stimulus (Jacob et al., 2013). Other works provide evidence that ouabain also inhibits neutrophil transmigration into lung tissue in mice models of inflammatory allergy (Galvão et al., 2017) and acute pulmonary injury (Wang et al., 2018). In vitro studies also presented the inhibitory effect of ouabain on rabbit (Ward and Becker, 1970), human (Ray and Samanta, 1997), and mice neutrophil chemotaxis (Cavalcante-Silva et al., 2021a). The cardiotonic steroid marinobufagenin also reduces neutrophil migration during inflammation, corroborating the ouabain effect (Carvalho et al., 2019) (Figure 1B).

The exact mechanism of action of ouabain in impairment neutrophil migration remains to be fully elucidated, however, some evidence is emerging. Chemoattractant gradients trigger neutrophils intracellular signaling that guides these cells towards inflammatory tissues. The MAPK signaling mediates neutrophils chemotaxis (Liew and Kubes, 2019). It was observed that ouabain reduces p38 phosphorylation, but not ERK activation in mice neutrophils (Cavalcante-Silva L. H. A. et al., 2021). Neutrophil's receptors, including adhesion molecules, can be regulated by p38 MAPKdependent signaling (Kim and Haynes, 2013). Indeed, ouabain can reduce $\alpha$ (Ninsontia and Chanvorachote, 2014) and $\beta$ (Cavalcante-Silva et al., 2020) integrins in different types of cells, nevertheless the real impact of this effects in neutrophil migration should still be addressed. On the other hand, ouabain does not reduce the chemokine receptor CXCR2 expression in mice neutrophils or modulates the levels of its ligands CXCL1 (Cavalcante-Silva et al., 2020). However, in human neutrophils, ouabain interferes with chemokine receptor (CXCR1/2) recycling, which in turns decreases neutrophil migration (Ray and Samanta, 1997).

Additionally, in models of peritoneal (Leite et al., 2015) and pulmonary (Wang et al., 2018) inflammation, ouabain also inhibits NF- $\kappa \mathrm{B}$ pathway. The activation of this transcription factor is associated with release of proinflammatory cytokines, which in turn stimulates endothelial and immune cells (Netea et al., 2017). In fact, ouabain reduces TNF- $\alpha$ and IL- $\beta$ release (Leite et al., 2015), therefore this may be associated with impaired migration of neutrophils. Moreover, in A549 cells, this steroid decreases the TNF- $\alpha$-induced expression of ICAM1 , an adhesion molecule that binds integrin (Takada et al., 2009).

The mechanisms used by neutrophils during an immune response include phagocytosis, NETs, formation of reactive oxygen species and release of microbicidal molecules contained in cytoplasmic granules (i.e., myeloperoxidase, neutrophilic elastase, and others) (Yang et al., 2017). Neutrophils also produce different cytokines (eg, IL-1Ra, IL-12, IL-23, TNF- $\alpha$, G-CSF, among others) and chemokines (eg, CXCL-1, CCL-20, CCL-2, among others) that modulate the immune response (Mantovani et al., 2011; Yang et al., 2017; Burn et al., 2021). In human neutrophils, it has been shown that ouabain at $100 \mathrm{nM}$ induces DNA release, without promote necrosis, suggesting NETs release (Silva et al., 2021). Additionally, in rat neutrophils, ouabain reduces generation of free radical induced by NO donors, this effect could be related to membrane depolarization (Patel et al., 2009).

Although studies have shown that ouabain interferes with neutrophil infiltration induced by different stimuli in the periphery, such as concanavalin A (de Vasconcelos et al., 2011), Zimosan (Leite et al., 2015), ovalbumin (Galvão et al., 2017) and Leishmania amazonensis (Jacob et al., 2013), as well as the activation of these cells, since ouabain modulate the generation of free radicals induced by nitric oxide donors (Patel et al., 2009) and the release of NETs (Silva et al., al, 2021), studies lack data about ouabain modulation on neutrophils in the neuroinflammation; however, a study observed the presence of neutrophils in a model of ouabain-induced injury in zebrafish. Mitchell et al. (2018) 
observed that the retinal lesion resulting from the administration of ouabain was accompanied by an early leukocyte infiltration, followed by a period in which there was proliferation of immune cells, possibly from the resident microglia and macrophages derived from extra-retinal regions. Furthermore, the presence of neutrophils was observed in the vitreous leukocyte population, identified by the expression of myeloid-specific peroxidase $(\mathrm{mpx})+$, supporting that retinal injury induced by ouabain is accompanied by an early migration of leukocytes from the bloodstream. After 24, 48 and $72 \mathrm{~h}$ of ouabain administration, very few $\mathrm{mpx}+$ neutrophils were identified, suggesting that there was no significant increase in the number of neutrophils at these times (Mitchell et al., 2018).

Despite there is no compelling data on the effects of ouabain and its receptor, NKA, on neutrophil dynamics in neuroinflammatory diseases, robust studies point to the importance of ouabain-NKA signaling in neuroinflammation (reviewed in Orellana et al., 2016; Leite et al., 2020). In addition, the immunomodulatory role of ouabain in neutrophil dynamics has been observed in classical models of peripheral inflammation (Leite et al., 2015; Cavalcante-Silva et al., 2020). These data together suggest that ouabain-NKA signaling may be an important marker to be investigated in neutrophil dynamics in neuroinflammatory diseases, thus favoring a better understanding of the pathophysiology involved in the progression of these diseases, as well as aiding in the discovery of new strategies for neurodegenerative diseases.

\section{REFERENCES}

Akira, S., Uematsu, S., and Takeuchi, O. (2006). Pathogen Recognition and Innate Immunity. Cell 124, 783-801. doi:10.1016/J.CELL.2006.02.015

Alpert, J. S. (2021). Is Digitalis Therapy Still Viable? Foxglove Therapy Makes a Comeback. Am. J. Med. 134, 1-2. doi:10.1016/J.AMJMED.2020.09.001

Bagrov, A. Y., Shapiro, J. I., and Fedorova, O. V. (2009). Endogenous Cardiotonic Steroids: Physiology, Pharmacology, and Novel Therapeutic Targets. Pharmacol. Rev. 61, 9-38. doi:10.1124/pr.108.000711

Bautista-Becerril, B., Campi-Caballero, R., Sevilla-Fuentes, S., Hernández-Regino, L. M., Hanono, A., Flores-Bustamante, A., et al. (2021). Immunothrombosis in Covid-19: Implications of Neutrophil Extracellular Traps. Biomolecules 11. 694. doi:10.3390/biom11050694

Blanco, G., and Mercer, R. W. (1998). Isozymes of the Na-K-ATPase: Heterogeneity in Structure, Diversity in Function. Am. J. Physiol. 275, F633-F650. doi:10.1152/AJPRENAL.1998.275.5.F633

Blaustein, M. P., and Hamlyn, J. M. (2020). Ouabain, Endogenous Ouabain and Ouabain-like Factors: The Na+ Pump/ouabain Receptor, its Linkage to NCX, and its Myriad Functions. Cell Calcium 86, 102159. doi:10.1016/j.ceca.2020. 102159

Burn, G. L., Foti, A., Marsman, G., Patel, D. F., and Zychlinsky, A. (2021). The Neutrophil. Immunity 54, 1377-1391. doi:10.1016/J.IMMUNI.2021.06.006

Carvalho, D. C. M., Cavalcante-Silva, L. H. A., Lima, É. A., Galvão, J. G. F. M., Alves, A. K. A., Feijó, P. R. O., et al. (2019). Marinobufagenin Inhibits Neutrophil Migration and Proinflammatory Cytokines. J. Immunol. Res. 2019, 1-11. doi:10.1155/2019/1094520

Cavalcante-Silva, L. H. A., Carvalho, D. C. M., Lima, É. A., Galvão, J. G. F. M., da Silva, J. S. F., Sales-Neto, J. M., et al. (2021b). Neutrophils and COVID-19: The Road So Far. Int. Immunopharmacol. 90, 107233. doi:10.1016/j.intimp.2020. 107233

\section{AUTHOR CONTRIBUTIONS}

Elaborated the figure and wrote the manuscript: JL, LC-S, MR, and GdM. Reviewed topics and concepts: CS and SR-M. Conceived, reviewed and discussed concepts in the manuscript: CS and SR-M. All authors contributed to the article and approved the submitted version.

\section{FUNDING}

This publication was made possible by grants from São Paulo Research Foundation (FAPESP) (2016/07427-8); National Council for Scientific and Technological Development $(\mathrm{CNPq}$ 405089/2018-0); and Coordenação de Aperfeiçoamento de Pessoal de Nível SuperiorCAPES (CAPES-STINT program 88887.125409/2016-00-Joint Brazilian-Swedish Research Collaboration) and USP Neuroscience Research Support Centres (NAPNA) to CS. JL was a research fellow from FAPESP, and MR, and GdM are Ph.D. fellowship from CNPq; $\mathrm{CS}$ and SR-M are research fellows of CNPq.

\section{ACKNOWLEDGMENTS}

We thank Elsevier Author Service for English Editing, and Larissa de Sá Lima for technical support. Figure 1 was created with BioRender.com.

Cavalcante-Silva, L. H. A., Lima, É. A., Carvalho, D. C. M., de Sales-Neto, J. M., Alves, A. K. A., Galvão, J. G. F. M., et al. (2017). Much More Than a Cardiotonic Steroid: Modulation of Inflammation by Ouabain. Front. Physiol. 8, 895. doi:10. 3389/fphys.2017.00895

Cavalcante-Silva, L. H. A., Lima, É. A., Carvalho, D. C. M., and RodriguesMascarenhas, S. (2020). Ouabain Reduces the Expression of the Adhesion Molecule CD18 in Neutrophils. Inflammopharmacology 28, 787-793. doi:10. 1007/s10787-019-00602-8

Cavalcante-Silva, L. H. A., Carvalho, D. C. M., de Almeida Lima, É., and RodriguesMascarenhas, S. (2021a). Ouabain Inhibits P38 Activation in Mice Neutrophils. Inflammopharmacol 29, 1829-1833. doi:10.1007/S10787-021-00882-Z

Chen, Y., Huang, W., Yang, M., Xin, G., Cui, W., Xie, Z., et al. (2017). Cardiotonic Steroids Stimulate Macrophage Inflammatory Responses through a Pathway Involving CD36, TLR4, and Na/K-ATPase. Arterioscler. Thromb. Vasc. Biol. 37, 1462-1469. doi:10.1161/ATVBAHA.117.309444

Christy, A. L., Walker, M. E., Hessner, M. J., and Brown, M. A. (2013). Mast Cell Activation and Neutrophil Recruitment Promotes Early and Robust Inflammation in the Meninges in EAE. J. Autoimmun. 42, 50-61. doi:10.1016/J.JAUT.2012.11.003

Cuartero, M. I., Ballesteros, I., Moraga, A., Nombela, F., Vivancos, J., Hamilton, J. A., et al. (2013). N2 Neutrophils, Novel Players in Brain Inflammation after Stroke: Modulation by the PPAR $\gamma$ Agonist Rosiglitazone. Stroke 44, 3498-3508. doi:10.1161/STROKEAHA.113.002470

Cui, X., and Xie, Z. (2017). Protein Interaction and Na/K-ATPase-Mediated Signal Transduction. Molecules 22. doi:10.3390/molecules22060990

da Silva, J. M., das Neves Azevedo, A., dos Santos Barbosa, R. P., Vianna, T. A., Fittipaldi, J., Teixeira, M. P., et al. (2016). Dynamics of Murine B Lymphocytes Is Modulated by In Vivo Treatment with Steroid Ouabain. Immunobiology 221, 368-376. doi:10.1016/j.imbio.2015.09.020

de Paiva, L. S., Costa, K. M., Canto, F. B., Cabral, V. R., Fucs, R., Nobrega, A., et al. (2011). Modulation of Mature B Cells in Mice Following Treatment with Ouabain. Immunobiology 216, 1038-1043. doi:10.1016/j.imbio.2011.03.002 
de Vasconcelos, D. I. B., Leite, J. A., Carneiro, L. T., Piuvezam, M. R., de Lima, M. R. V., de Morais, L. C. L., et al. (2011). Anti-inflammatory and Antinociceptive Activity of Ouabain in Mice. Mediators Inflamm. 2011, 1-11. doi:10.1155/2011/ 912925

Dinkel, K., Dhabhar, F. S., and Sapolsky, R. M. (2004). Neurotoxic Effects of Polymorphonuclear Granulocytes on Hippocampal Primary Cultures. Proc. Natl. Acad. Sci. U. S. A. 101, 331-336. doi:10.1073/PNAS.0303510101

Doty, K. R., Guillot-Sestier, M. V., and Town, T. (2015). The Role of the Immune System in Neurodegenerative Disorders: Adaptive or Maladaptive. Brain Res. 1617, 155-173. doi:10.1016/J.BRAINRES.2014.09.008

Du, J., Jiang, L., Chen, F., Hu, H., and Zhou, M. (2021). Cardiac Glycoside Ouabain Exerts Anticancer Activity via Downregulation of STAT3. Front. Oncol. 11. 1275. doi:10.3389/FONC.2021.684316

Dvela, M., Rosen, H., Feldmann, T., Nesher, M., and Lichtstein, D. (2007). Diverse Biological Responses to Different Cardiotonic Steroids. Pathophysiology 14, 159-166. doi:10.1016/J.PATHOPHYS.2007.09.011

Ellman, D. G., Isaksen, T. J., Lund, M. C., Dursun, S., Wirenfeldt, M., Jørgensen, L. H., et al. (2017). The Loss-Of-Function Disease-Mutation G301R in the $\mathrm{Na}+$ / $\mathrm{K}+\mathrm{ATPase}$ a2 Isoform Decreases Lesion Volume and Improves Functional Outcome after Acute Spinal Cord Injury in Mice. BMC Neurosci. 18, 66. doi:10. 1186/S12868-017-0385-9

Fan, X., Xie, J., and Tian, J. (2017). Reducing Cardiac Fibrosis: Na/K-ATPase Signaling Complex as a Novel Target. Cardiovasc. Pharm. Open Access 6. 204. doi:10.4172/2329-6607.1000204

Ferrandi, M., Manunta, P., Balzan, S., Hamlyn, J. M., Bianchi, G., and Ferrari, P. (1997). Ouabain-like Factor Quantification in Mammalian Tissues and Plasma: Comparison of Two Independent Assays. Hypertension 30, 886-896. doi:10. 1161/01.HYP.30.4.886

Foey, A. D., Crawford, A., and Hall, N. D. (1997). Modulation of Cytokine Production by Human Mononuclear Cells Following Impairment of $\mathrm{Na}$, K-ATPase Activity. Biochim. Biophys. Acta 1355, 43-49. doi:10.1016/S01674889(96)00116-4

Forshammar, J., Block, L., Lundborg, C., Biber, B., and Hansson, E. (2011). Naloxone and Ouabain in Ultralow Concentrations Restore $\mathrm{Na}+\mathrm{K}+-$ ATPase and Cytoskeleton in Lipopolysaccharide-Treated Astrocytes. J. Biol. Chem. 286, 31586-31597. doi:10.1074/JBC.M111.247767

Forshammar, J., Jörneberg, P., Björklund, U., Westerlund, A., Lundborg, C., Biber, B., et al. (2013). Anti-inflammatory Substances Can Influence Some Glial Cell Types but Not Others. Brain Res. 1539, 34-40. doi:10.1016/J.BRAINRES.2013. 09.052

Gallardo, G., Barowski, J., Ravits, J., Siddique, T., Lingrel, J. B., Robertson, J., et al. (2014). An $\alpha 2-\mathrm{Na} / \mathrm{K}$ ATPase/a-Adducin Complex in Astrocytes Triggers Noncell Autonomous Neurodegeneration. Nat. Neurosci. 17, 1710-1719. doi:10. 1038/NN.3853

Galvão, J. G. F. M., Cavalcante-Silva, L. H. A., Carvalho, D. C. M., Ferreira, L. K. D. P., Monteiro, T. M., Alves, A. F., et al. (2017). Ouabain Attenuates OvalbuminInduced Airway Inflammation. Inflamm. Res. 66, 1117-1130. doi:10.1007/ s00011-017-1092-9

García-Culebras, A., Durán-Laforet, V., Peña-Martínez, C., Moraga, A., Ballesteros, I., Cuartero, M. I., et al. (2019). Role of TLR4 (Toll-like Receptor 4) in N1/N2 Neutrophil Programming after Stroke. Stroke 50, 2922-2932. doi:10.1161/ STROKEAHA.119.025085

Gonçalves-de-Albuquerque, C. F., Burth, P., Silva, A. R., de Moraes, I. M., Oliveira, F. M., Santelli, R. E., et al. (2014). Murine Lung Injury Caused by Leptospira Interrogans Glycolipoprotein, a Specific Na/K-ATPase Inhibitor. Respir. Res. 15, 93. doi:10.1186/s12931-014-0093-2

Hamlyn, J. M., Blaustein, M. P., Bova, S., DuCharme, D. W., Harris, D. W., Mandel, F., et al. (1991). Identification and Characterization of a Ouabain-like Compound from Human Plasma. Proc. Natl. Acad. Sci. U. S. A. 88, 6259-6263. Available at:http://www.ncbi.nlm.nih.gov/pubmed/1648735 (Accessed December 13, 2018). doi:10.1073/pnas.88.14.6259

Hawkes, C. A., and McLaurin, J. (2009). Selective Targeting of Perivascular Macrophages for Clearance of Beta-Amyloid in Cerebral Amyloid Angiopathy. Proc. Natl. Acad. Sci. U. S. A. 106, 1261-1266. doi:10.1073/ PNAS.0805453106

Hellebrekers, P., Vrisekoop, N., and Koenderman, L. (2018). Neutrophil Phenotypes in Health and Disease. Eur. J. Clin. Invest. 48 Suppl 2, e12943. doi:10.1111/eci.12943
Herz, J., Sabellek, P., Lane, T. E., Gunzer, M., Hermann, D. M., and Doeppner, T. R. (2015). Role of Neutrophils in Exacerbation of Brain Injury after Focal Cerebral Ischemia in Hyperlipidemic Mice. Stroke 46, 2916-2925. doi:10.1161/ STROKEAHA.115.010620

Hidalgo, A., Chilvers, E. R., Summers, C., and Koenderman, L. (2019). The Neutrophil Life Cycle. Trends Immunol. 40, 584-597. doi:10.1016/j.it.2019. 04.013

Hou, Y., Yang, D., Xiang, R., Wang, H., Wang, X., Zhang, H., et al. (2019). N2 Neutrophils May Participate in Spontaneous Recovery after Transient Cerebral Ischemia by Inhibiting Ischemic Neuron Injury in Rats. Int. Immunopharmacol. 77, 105970. doi:10.1016/J.INTIMP.2019.105970

Huh, J. R., Leung, M. W., Huang, P., Ryan, D. A., Krout, M. R., Malapaka, R. R., et al. (2011). Digoxin and its Derivatives Suppress TH17 Cell Differentiation by Antagonizing ROR $\gamma$ t Activity. Nature 472, 486-490. doi:10.1038/nature09978

Isaksen, T. J., and Lykke-Hartmann, K. (2016). Insights into the Pathology of the a2- $\mathrm{Na}(+) / \mathrm{K}(+)$-ATPase in Neurological Disorders; Lessons from Animal Models. Front. Physiol. 7, 161. doi:10.3389/FPHYS.2016.00161

Jacob, P. L., Leite, J. A., Alves, A. K., Rodrigues, Y. K., Amorim, F. M., Néris, P. L., et al. (2013). Immunomodulatory Activity of Ouabain in Leishmania Leishmania Amazonensis-Infected Swiss Mice. Parasitol. Res. 112, 1313-1321. doi:10.1007/s00436-012-3146-9

Jones, H. R., Robb, C. T., Perretti, M., and Rossi, A. G. (2016). The Role of Neutrophils in Inflammation Resolution. Semin. Immunol. 28, 137-145. doi:10. 1016/j.smim.2016.03.007

Kanashiro, A., Hiroki, C. H., da Fonseca, D. M., Birbrair, A., Ferreira, R. G., Bassi, G. S., et al. (2020). The Role of Neutrophils in Neuro-Immune Modulation. Pharmacol. Res. 151, 104580. doi:10.1016/J.PHRS.2019.104580

Kang, J., Jiang, M. H., Min, H. J., Jo, E. K., Lee, S., Karin, M., et al. (2011). IKK- $\beta$ mediated Myeloid Cell Activation Exacerbates Inflammation and Inhibits Recovery after Spinal Cord Injury. Eur. J. Immunol. 41, 1266-1277. doi:10. 1002/EJI.201040582

Kennedy, D. J., Chen, Y., Huang, W., Viterna, J., Liu, J., Westfall, K., et al. (20131979). CD36 and Na/K-ATPase-A1 Form a Proinflammatory Signaling Loop in Kidney. Hypertension 61, 216-224. doi:10.1161/ HYPERTENSIONAHA.112.198770

Kim, D., and Haynes, C. L. (2013). The Role of P38 MAPK in Neutrophil Functions: Single Cell Chemotaxis and Surface Marker Expression. Analyst 138, 6826-6833. doi:10.1039/c3an01076g

Kinoshita, P. F., Yshii, L. M., Orellana, A. M. M., Paixão, A. G., Vasconcelos, A. R., Lima, L. S., et al. (2017). Alpha $2 \mathrm{Na}+\mathrm{K}+-\mathrm{ATPase}$ Silencing Induces Loss of Inflammatory Response and Ouabain protection in Glial Cells. Sci. Rep. 7, 4894. doi:10.1038/s41598-017-05075-9

Kinoshita, P. F., Yshii, L. M., Vasconcelos, A. R., Orellana, A. M., Lima, Lde. S., Davel, A. P., et al. (2014). Signaling Function of Na,K-ATPase Induced by Ouabain against LPS as an Inflammation Model in hippocampus. J. Neuroinflammation 11, 218. doi:10.1186/s12974-014-0218-z

Kolaczkowska, E., and Kubes, P. (2013). Neutrophil Recruitment and Function in Health and Inflammation. Nat. Rev. Immunol. 13, 159-175. doi:10.1038/ nri3399

Lampron, A., Pimentel-Coelho, P. M., and Rivest, S. (2013). Migration of Bone Marrow-Derived Cells into the central Nervous System in Models of Neurodegeneration. J. Comp. Neurol. 521, 3863-3876. doi:10.1002/CNE.23363

Lancaster, M. C., and Vegad, J. L. (1967). Suppression of the Early Inflammatory Response in the Sheep by Strophanthin G. Nature 213, 840-841. doi:10.1038/ $213840 \mathrm{~b} 0$

Laredo, J., Hamilton, B. P., and Hamlyn, J. M. (1994). Ouabain Is Secreted by Bovine Adrenocortical Cells. Endocrinology 135, 794-797. doi:10.1210/endo. 135.2.8033829

Laredo, J., Hamilton, B. P., and Hamlyn, J. M. (1995). Secretion of Endogenous Ouabain from Bovine Adrenocortical Cells: Role of the Zona Glomerulosa and Zona Fasciculata. Biochem. Biophys. Res. Commun. 212, 487-493. doi:10.1006/ bbrc.1995.1996

Leite, J. A., Alves, A. K., Galvão, J. G., Teixeira, M. P., Cavalcante-Silva, L. H., Scavone, C., et al. (2015). Ouabain Modulates Zymosan-Induced Peritonitis in Mice. Mediators Inflamm. 2015, 1-12. doi:10.1155/2015/265798

Leite, J. A., Isaksen, T. J., Heuck, A., Scavone, C., and Lykke-Hartmann, K. (2020). The $\alpha 2 \mathrm{Na}+/ \mathrm{K}+$-ATPase Isoform Mediates LPS-Induced Neuroinflammation. Sci. Rep. 10, 14180. doi:10.1038/S41598-020-71027-5 
Leliefeld, P. H., Wessels, C. M., Leenen, L. P., Koenderman, L., and Pillay, J. (2016). The Role of Neutrophils in Immune Dysfunction during Severe Inflammation. Crit. Care 20, 73. doi:10.1186/s13054-016-1250-4

Liew, P. X., and Kubes, P. (2019). The Neutrophil's Role during Health and Disease. Physiol. Rev. 99, 1223-1248. doi:10.1152/physrev.00012.2018

Liu, J., Tian, J., Haas, M., Shapiro, J. I., Askari, A., and Xie, Z. (2000). Ouabain Interaction with Cardiac $\mathrm{Na}+/ \mathrm{K}+$-ATPase Initiates Signal Cascades Independent of Changes in Intracellular $\mathrm{Na}+$ and $\mathrm{Ca} 2+$ Concentrations. J. Biol. Chem. 275, 27838-27844. doi:10.1074/jbc.M002950200

Liu, J., and Xie, Z. J. (2010). The Sodium Pump and Cardiotonic Steroids-Induced Signal Transduction Protein Kinases and Calcium-Signaling Microdomain in Regulation of Transporter Trafficking. Biochim. Biophys. Acta 1802, 1237-1245. doi:10.1016/j.bbadis.2010.01.013

Mantovani, A., Cassatella, M. A., Costantini, C., and Jaillon, S. (2011). Neutrophils in the Activation and Regulation of Innate and Adaptive Immunity. Nat. Rev. Immunol. 11, 519-531. doi:10.1038/nri3024

Markov, A. G., Fedorova, A. A., Kravtsova, V. V., Bikmurzina, A. E., Okorokova, L. S., Matchkov, V. V., et al. (2020). Circulating Ouabain Modulates Expression of Claudins in Rat Intestine and Cerebral Blood Vessels. Int. J. Mol. Sci. 21, 1-16. doi:10.3390/IJMS21145067

Matozzo, F. H., Votto, A. P. S., Rodrigues-Mascarenhas, S., Cavalcante-Silva, L. H. A., Valente, R. C., and Rumjanek, V. M. (2020). Ouabain as an Anti-cancer Agent. Curr. Top. Biochem. Res. 21, 25-40. Available at: http://www. researchtrends.net $/$ tia/abstract.asp? $\mathrm{in}=0 \& \mathrm{vn}=21 \& \mathrm{tid}=40 \& \mathrm{aid}=6579 \mathrm{\& pub}=$ 2020\&type $=3$ (Accessed January 9, 2022).

Matsumori, A., Ono, K., Nishio, R., Igata, H., Shioi, T., Matsui, S., et al. (1997). Modulation of Cytokine Production and protection against Lethal Endotoxemia by the Cardiac Glycoside Ouabain. Circulation 96, 1501-1506. doi:10.1161/01.CIR.96.5.1501

Mázala-de-Oliveira, T., de Figueiredo, C. S., de Rezende Corrêa, G., da Silva, M. S., Miranda, R. L., de Azevedo, M. A., et al. (2021). Ouabain-Na+/K+-ATPase Signaling Regulates Retinal Neuroinflammation and ROS Production Preventing Neuronal Death by an Autophagy-dependent Mechanism Following Optic Nerve Axotomy In Vitro. Neurochem. Res. 2021, 1-16. doi:10.1007/S11064-021-03481-0

Minns, D., Smith, K. J., and Findlay, E. G. (2019). Orchestration of Adaptive T Cell Responses by Neutrophil Granule Contents. Mediators Inflamm. 2019, 1-15. doi:10.1155/2019/8968943

Mishalian, I., Granot, Z., and Fridlender, Z. G. (2017). The Diversity of Circulating Neutrophils in Cancer. Immunobiology 222, 82-88. doi:10.1016/j.imbio.2016. 02.001

Mitchell, D. M., Lovel, A. G., and Stenkamp, D. L. (2018). Dynamic Changes in Microglial and Macrophage Characteristics during Degeneration and Regeneration of the Zebrafish Retina. J. Neuroinflammation 15, 163. doi:10. 1186/S12974-018-1185-6

Nascimento, C. R., Valente, R. C., Echevarria-Lima, J., Fontes, C. F. L., de AraujoMartins, L., Araujo, E. G., et al. (2014). The Influence of Ouabain on Human Dendritic Cells Maturation. Mediators Inflamm. 2014, 1-15. doi:10.1155/2014/ 494956

Netea, M. G., Balkwill, F., Chonchol, M., Cominelli, F., Donath, M. Y., GiamarellosBourboulis, E. J., et al. (2017). A Guiding Map for Inflammation. Nat. Immunol. 18, 826-831. doi:10.1038/NI.3790

Neumann, J., Riek-Burchardt, M., Herz, J., Doeppner, T. R., König, R., Hütten, H., et al. (2015). Very-late-antigen-4 (VLA-4)-Mediated Brain Invasion by Neutrophils Leads to Interactions with Microglia, Increased Ischemic Injury and Impaired Behavior in Experimental Stroke. Acta Neuropathol. 129, 259-277. doi:10.1007/S00401-014-1355-2

Nguyen, H. X., O'Barr, T. J., and Anderson, A. J. (2007). Polymorphonuclear Leukocytes Promote Neurotoxicity through Release of Matrix Metalloproteinases, Reactive Oxygen Species, and TNF-Alpha. J. Neurochem. 102, 900-912. doi:10.1111/J.1471-4159.2007.04643.X

Ninsontia, C., and Chanvorachote, P. (2014). Ouabain Mediates Integrin Switch in Human Lung Cancer Cells. Anticancer Res. 34, 5495-5502. Available at: http://www.ncbi.nlm.nih.gov/pubmed/25275046 (Accessed December 2, 2017).

Nourshargh, S., and Alon, R. (2014). Leukocyte Migration into Inflamed Tissues. Immunity 41, 694-707. doi:10.1016/J.IMMUNI.2014.10.008
Ocana, A., Nieto-Jiménez, C., Pandiella, A., and Templeton, A. J. (2017). Neutrophils in Cancer: Prognostic Role and Therapeutic Strategies. Mol. Cancer 16, 137. doi:10.1186/s12943-017-0707-7

Okazaki, T., Ilea, V. S., Okazaki, A., Wicher, K., Reisman, R. E., and Arbesman, C. E. (1976). Inhibition of Antigen-Induced Histamine Release by Ouabain. J. Allergy Clin. Immunol. 57, 454-462. doi:10.1016/0091-6749(76)90061-0

Olej, B., de La Rocque, L., Castilho, F. P., Mediano, I. F., Campos, M. M., and Rumjanek, V. M. (1994). Effect of Ouabain on Lymphokine-Activated Killer Cells. Int. J. Immunopharmacol. 16, 769-774. doi:10.1016/0192-0561(94)90097-3

O'Neil, L. J., and Kaplan, M. J. (2019). Neutrophils in Rheumatoid Arthritis: Breaking Immune Tolerance and Fueling Disease. Trends Mol. Med. 25, 215-227. doi:10.1016/J.MOLMED.2018.12.008

Orellana, A. M., Kinoshita, P. F., Leite, J. A., Kawamoto, E. M., and Scavone, C. (2016). Cardiotonic Steroids as Modulators of Neuroinflammation. Front. Endocrinol. (Lausanne) 7, 10. doi:10.3389/fendo.2016.00010

Pamnani, M. B., Buggy, J., Huot, S. J., and Haddy, F. J. (1981). Studies on the Role of a Humoral Sodium-Transport Inhibitor and the Anteroventral Third Ventricle (AV3V) in Experimental Low-Renin Hypertension. Clin. Sci. (Lond) 61 Suppl 7, 57s-60s. doi:10.1042/CS061057S

Parackova, Z., Bloomfield, M., Klocperk, A., and Sediva, A. (2021). Neutrophils Mediate Th17 Promotion in COVID-19 Patients. J. Leukoc. Biol. 109, 73-76. doi:10.1002/JLB.4COVCRA0820-481RRR

Patel, S., Vemula, J., Konikkat, S., Barthwal, M. K., and Dikshit, M. (2009). Ion Channel Modulators Mediated Alterations in NO-Induced Free Radical Generation and Neutrophil Membrane Potential. Free Radic. Res. 43, 514-521. doi:10.1080/10715760902887276

Phillipson, M., and Kubes, P. (2019). The Healing Power of Neutrophils. Trends Immunol. 40, 635-647. doi:10.1016/j.it.2019.05.001

Pierson, E. R., Wagner, C. A., and Goverman, J. M. (2018). The Contribution of Neutrophils to CNS Autoimmunity. Clin. Immunol. 189, 23-28. doi:10.1016/J. CLIM.2016.06.017

Ray, E., and Samanta, A. K. (1997). Receptor-mediated Endocytosis of IL-8: a Fluorescent Microscopic Evidence and Implication of the Process in LigandInduced Biological Response in Human Neutrophils. Cytokine 9, 587-596. doi:10.1006/cyto.1997.0206

Rodrigues Mascarenhas, S., Echevarria-Lima, J., Fernandes dos Santos, N., and Rumjanek, V. M. (2003). CD69 Expression Induced by Thapsigargin, Phorbol Ester and Ouabain on Thymocytes Is Dependent on External Ca2+ Entry. Life Sci. 73, 1037-1051. Available at: http://www.ncbi.nlm.nih.gov/pubmed/ 12818356 (Accessed November 27, 2017). doi:10.1016/s0024-3205(03)00377-1

Rodrigues-Mascarenhas, S., Bloise, F. F., Moscat, J., and Rumjanek, V. M. (2008). Ouabain Inhibits P38 Activation in Thymocytes. Cell Biol. Int. 32, 1323-1328. doi:10.1016/j.cellbi.2008.07.012

Rodrigues-Mascarenhas, S., Da Silva de Oliveira, A., Amoedo, N. D., AffonsoMitidieri, O. R., Rumjanek, F. D., and Rumjanek, V. M. (2009). Modulation of the Immune System by Ouabain. Ann. N. Y. Acad. Sci. 1153, 153-163. doi:10. 1111/j.1749-6632.2008.03969.x

Sas, A. R., Carbajal, K. S., Jerome, A. D., Menon, R., Yoon, C., Kalinski, A. L., et al. (2020). A New Neutrophil Subset Promotes CNS Neuron Survival and Axon Regeneration. Nat. Immunol. 21, 1496-1505. doi:10.1038/S41590-020-00813-0

Schneider, N. F. Z., Cerella, C., Simões, C. M. O., and Diederich, M. (2017). Anticancer and Immunogenic Properties of Cardiac Glycosides. Molecules 22. 1932. doi:10.3390/MOLECULES22111932

Silva, L., Carvalho, D., Lima, E., Nadaes, N., Saraiva, E., and Mascarenhas, S. (2021). Ouabain Triggers Neutrophil Extracellular Traps in Human Neutrophils.6th European Congress of Immunology, 1-4 September 2021, Virtual meeting: European Journal of Immunology: vol 51, No S1, Available at: https:// onlinelibrary.wiley.com/toc/15214141/2021/51/S1 (Accessed November 13, 2021).

Simmons, S. B., Liggitt, D., and Goverman, J. M. (2014). Cytokine-regulated Neutrophil Recruitment Is Required for Brain but Not Spinal Cord Inflammation during Experimental Autoimmune Encephalomyelitis. J. Immunol. 193, 555-563. doi:10.4049/JIMMUNOL.1400807

Škubník, J., Pavlíčková, V., Rimpelová, S., Atkin-Smith, G., Tixeira, R., and Baxter, A. A. (2021). Cardiac Glycosides as Immune System Modulators. Biomolecules 11, 659. doi:10.3390/BIOM11050659

Smolyaninova, L. V., Dergalev, A. A., Kulebyakin, K. Y., Carpenter, D. O., and Boldyrev, A. A. (2013). Carnosine Prevents Necrotic and Apoptotic Death of 
Rat Thymocytes via Ouabain-Sensitive Na/K-ATPase. Cell Biochem. Funct. 31, 30-35. doi:10.1002/cbf.2856

Stock, A. J., Kasus-Jacobi, A., and Pereira, H. A. (2018). The Role of Neutrophil Granule Proteins in Neuroinflammation and Alzheimer's Disease. J. Neuroinflammation 15, 240. doi:10.1186/S12974-018-1284-4

Szamel, M., Schneider, S., and Resch, K. (1981). Functional Interrelationship between $(\mathrm{Na}++\mathrm{K}+)$-ATPase and Lysolecithin Acyltransferase in Plasma Membranes of Mitogen-Stimulated Rabbit Thymocytes. J. Biol. Chem. 256, 9198-9204. Available at: http://www.ncbi.nlm.nih.gov/pubmed/6267065 (Accessed January 16, 2020). doi:10.1016/s0021-9258(19)52528-5

Takada, Y., Matsuo, K., Ogura, H., Bai, L., Toki, A., Wang, L., et al. (2009). Odoroside $\mathrm{A}$ and Ouabain Inhibit $\mathrm{Na}+\mathrm{K}+-\mathrm{ATPase}$ and Prevent NFkappaB-Inducible Protein Expression by Blocking $\mathrm{Na}$-dependent Amino Acid Transport. Biochem. Pharmacol. 78, 1157-1166. doi:10. 1016/j.bcp.2009.06.027

Teixeira, M. P., and Rumjanek, V. M. (2014). Ouabain Affects the Expression of Activation Markers, Cytokine Production, and Endocytosis of Human Monocytes. Mediators Inflamm. 2014, 760368. doi:10.1155/2014/760368

Valente, R. C., Nascimento, C. R., Araujo, E. G., and Rumjanek, V. M. (2009). mCD14 Expression in Human Monocytes Is Downregulated by Ouabain via Transactivation of Epithelial Growth Factor Receptor and Activation of P38 Mitogen-Activated Protein Kinase. Neuroimmunomodulation 16, 228-236. doi:10.1159/000212383

Wang, C., Meng, Y., Wang, Y., Jiang, Z., Xu, M., Bo, L., et al. (2018). Ouabain Protects Mice against Lipopolysaccharide-Induced Acute Lung Injury. Med. Sci. Monit. 24, 4455-4464. doi:10.12659/MSM.908627

Ward, P. A., and Becker, E. L. (1970). Potassium Reversible Inhibition of Leukotaxis by Ouabain. Life Sci. 9, 355-360. Available at: http://www.ncbi. nlm.nih.gov/pubmed/4315650 (Accessed December 1, 2017).
Xie, Z. (2003). Molecular Mechanisms of Na/K-ATPase-Mediated Signal Transduction. Ann. N.Y Acad. Sci. 986, 497-503. doi:10.1111/j.1749-6632.2003.tb07234.x

Yang, F., Feng, C., Zhang, X., Lu, J., and Zhao, Y. (2017). The Diverse Biological Functions of Neutrophils, beyond the Defense against Infections. Inflammation 40, 311-323. doi:10.1007/s10753-016-0458-4

Zenaro, E., Pietronigro, E., Bianca, V. D., Piacentino, G., Marongiu, L., Budui, S., et al. (2015). Neutrophils Promote Alzheimer's Disease-like Pathology and Cognitive Decline via LFA-1 Integrin. Nat. Med. 21, 880-886. doi:10.1038/NM.3913

Zhakeer, Z., Hadeer, M., Tuerxun, Z., and Tuerxun, K. (2017). Bufalin Inhibits the Inflammatory Effects in Asthmatic Mice through the Suppression of Nuclear Factor-Kappa B Activity. Pharmacology 99, 179-187. doi:10.1159/000450754

Conflict of Interest: The authors declare that the research was conducted in the absence of any commercial or financial relationships that could be construed as a potential conflict of interest.

Publisher's Note: All claims expressed in this article are solely those of the authors and do not necessarily represent those of their affiliated organizations, or those of the publisher, the editors, and the reviewers. Any product that may be evaluated in this article, or claim that may be made by its manufacturer, is not guaranteed or endorsed by the publisher.

Copyright $\odot 2022$ Leite, Cavalcante-Silva, Ribeiro, de Morais Lima, Scavone and Rodrigues-Mascarenhas. This is an open-access article distributed under the terms of the Creative Commons Attribution License (CC BY). The use, distribution or reproduction in other forums is permitted, provided the original author(s) and the copyright owner(s) are credited and that the original publication in this journal is cited, in accordance with accepted academic practice. No use, distribution or reproduction is permitted which does not comply with these terms. 